\title{
Extended quasiparticle approximation for relativistic electrons in plasmas
}

\author{
V.G.Morozov ${ }^{1}$, G.Röpke ${ }^{2}$ \\ ${ }^{1}$ Moscow State Institute of Radioengineering, Electronics and Automation, \\ 117454 Vernadsky Prospect 78, Moscow, Russia, \\ 2 Department of Physics, Rostock University, 18051 Rostock, Germany
}

Received April 17, 2006, in final form June 6, 2006

Starting with Dyson equations for the path-ordered Green's function, it is shown that the correlation functions for relativistic electrons (positrons) in a weakly coupled non-equilibrium plasmas can be decomposed into sharply peaked quasiparticle parts and off-shell parts in a rather general form. To leading order in the electromagnetic coupling constant, this decomposition yields the extended quasiparticle approximation for the correlation functions, which can be used for the first principle calculation of the radiation scattering rates in QED plasmas.

Key words: Many-particle QED, nonequilibrium Green's functions, kinetic theory of relativistic plasmas

PACS: 05.30.-d, 52.25.Dg, 52.27.-h, 52.27. $\mathrm{Ny}$

\section{Introduction}

There is an undeniable interest in studying QED phenomena in plasmas. Originally it was motivated by astrophysical problems, but recent progress in laser-plasma experiments shows that a significant number of particles in laboratory plasmas may have ultrarelativistic velocities. For instance, laser pulses with intensities $10^{19}-10^{20} \mathrm{~W} / \mathrm{cm}^{-2}$ are now available to drive intense electron beams with energies $10-300 \mathrm{MeV}[1,2]$.

Several difficulties are encountered when one attempts to consider QED processes in plasmas starting from first principles. Apart from the well-known vacuum divergencies and other field theoretical aspects, here appear problems which are characteristic of any statistical theory of nonequilibrium phenomena in many-particle systems. A general Green's function approach to QED plasmas was developed by Bezzerides and DuBois [3]. Within the lowest weak coupling approximation, they were able to derive a covariant particle kinetic equation including electron-electron (electronpositron) collisions and Cherenkov emission/absorption of plasmons. It should be noted, however, that the inclusion of radiative processes involving transverse photons (say, Bremsstrahlung, pair creation by hard photons, and Compton scattering) lies beyond the simplest pole (quasiparticle) approximation for the electron correlation functions used in [3]. The essential point is that in describing such processes, the off-shell parts of the field and particle correlation functions must be treated consistently. These terms describe virtual processes and contribute to the scattering rates. To solve the problem of off-shell contributions to the correlation functions, the so-called "extended quasiparticle approximation" was proposed in the context of the non-relativistic Green's function method $[4,5]$, where the off-shell corrections can be found to lowest order in the quasiparticle damping width. Unfortunately, this scheme becomes very complicated in the case of QED plasmas since one has to deal with matrix correlation functions and propagators in spinor space. A more general approach to the same problem was developed by Špička and Lipavský $[6,7]$. In principle, it allows one to go beyond the simplest version of the extended quasiparticle approximation by taking the collisional broadening of the quasiparticle spectral function into account. Another important point is that the main features of this approach are generalizable to relativistic systems. 
In this paper, following essentially the scheme of $[6,7]$, we formulate the extended quasiparticle representation for the correlation functions of relativistic electrons, which can serve as the first step toward Green's function theory of radiation processes in QED plasmas.

A few remarks about notation are appropriate here. We use the system of units with $c=$ $\hbar=1$ and the "rationalized" Lorentz-Heaviside units for electromagnetic field, i.e., the Coulomb interaction is written as $q q^{\prime} / 4 \pi r$. The signature of the metric tensor $g^{\mu \nu}$ is $(+,-,-,-)$. Cartesian components of three-dimensional vectors are denoted by subscripts: $V_{i}$, where $i=1,2,3$. With this convention, a four-vector has the components $V^{\mu}=\left(V^{0}, V_{i}\right)$ and $V_{\mu}=\left(V_{0},-V_{i}\right)$. For Dirac $\gamma$-matrices the common notation $\gamma^{\mu}=\left(\gamma^{0}, \gamma^{i}\right)$ is used. Summation over repeated spatial (Latin) and space-time (Greek) indices is implied. Our convention for the matrix Green's functions on the time-loop Schwinger-Keldysh contour follows Botermans and Malfliet [8].

\section{The electron Green's function in QED plasmas}

As is usually done in the standard Green's function formalism (see, e.g., [8,9]), we introduce the time-loop Schwinger-Keldysh contour $C$ shown in figure 1.

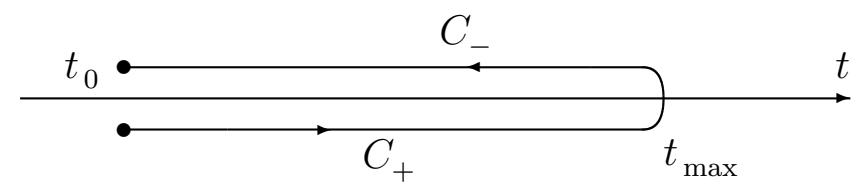

Figure 1. The time-loop contour $C$ with the chronological branch $\left(C_{+}\right)$and the anti-chronological branch $\left(C_{-}\right)$. In the following $t_{0} \rightarrow-\infty$ and $t_{\max } \rightarrow \infty$.

From now on, an underlined variable $(\underline{k})=\left(\underline{t}_{k}, \boldsymbol{r}_{k}\right)$ implies that $\underline{t}_{k}$ lies on the contour $C$, while the notation $(k)=\left(t_{k}, \boldsymbol{r}_{k}\right)$ is used for space-time variables. Integrals along $C$ are understood as

$$
\int_{C} \mathrm{~d} \underline{1} F(\underline{1})=\int_{-\infty}^{\infty} \mathrm{d} 1 F\left(1_{+}\right)-\int_{-\infty}^{\infty} \mathrm{d} 1 F\left(1_{-}\right),
$$

where $F\left(1_{ \pm}\right)$stands for functions with time arguments on the branches $C_{ \pm}$. For any function $F(\underline{1} 2)$ we introduce the canonical form [8]

$$
F(\underline{1} \underline{2})=\left(\begin{array}{cc}
F\left(1_{+} 2_{+}\right) & F\left(1_{+} 2_{-}\right) \\
F\left(12_{-}\right) & F\left(1_{-} 2_{-}\right)
\end{array}\right)=\left(\begin{array}{cc}
F^{<}+F^{+} & F^{<} \\
F^{>} & F^{<}-F^{-}
\end{array}\right)
$$

with space-time "correlation functions" $F^{\gtrless}(12)$ and retarded/advanced functions

$$
F^{ \pm}(12)=F_{0}(12) \delta\left(t_{1}-t_{2}\right) \pm \theta\left( \pm\left(t_{1}-t_{2}\right)\right)\left\{F^{>}(12)-F^{<}(12)\right\},
$$

where $F_{0}(12)$ is a singular contribution ${ }^{1}$, and $\theta(x)$ is the step function. Note the useful relation

$$
F^{>}(12)-F^{<}(12)=F^{+}(12)-F^{-}(12),
$$

which follows from the above definitions.

The electron (positron) Green's function in a QED plasma is defined as [3]

$$
G(\underline{1} \underline{2})=-\mathrm{i}\left\langle T_{C} \psi(\underline{1}) \bar{\psi}(\underline{2})\right\rangle,
$$

where $\psi(\underline{1})$ and $\bar{\psi}(\underline{1})=\psi^{\dagger}(\underline{1}) \gamma^{0}$ are Dirac fields. The path-ordering operator $T_{C}$ on the contour $C$ includes the usual sign convention for permutations of Fermi operators. Neglecting the effects of initial correlations which die out after a few collisions, the initial time $t_{0}$ in figure 1 will be taken

\footnotetext{
${ }^{1}$ In plasma physics, singular terms of this type enter the longitudinal field propagators [10].
} 
in the remote past, i.e., the limit $t_{0} \rightarrow-\infty$ will be assumed. In this case the ensemble average in (5) may be calculated with some initial density operator which admits Wick's decomposition [8].

Most applications of the Green's function method to plasma theory are based on the fact that $G(\underline{1} \underline{2})$ obeys Dyson equations [3]

$$
\begin{aligned}
& \left(\mathrm{i} \partial_{1}-e \not A(\underline{1})-m\right) G(\underline{1} \underline{2})=I \delta(\underline{1}-\underline{2})+\Sigma\left(\underline{1}^{\prime} \underline{1}^{\prime}\right) G\left(\underline{1}^{\prime} \underline{2}\right), \\
& G(\underline{1} \underline{2})\left(-\mathrm{i} \overleftarrow{\not}_{2}-e \not A(\underline{2})-m\right)=I \delta(\underline{1}-\underline{2})+G\left(\underline{1} \underline{1}^{\prime}\right) \Sigma\left(\underline{1}^{\prime} \underline{2}\right)
\end{aligned}
$$

where $A^{\mu}(\underline{1})=(\phi(\underline{1}), \boldsymbol{A}(\underline{1}))$ is the four-vector potential of the mean electromagnetic field, $I$ is the identity spinor matrix, $\delta(\underline{1}-\underline{2})$ is the delta function on the contour $C$, and $\Sigma(\underline{1} \underline{2})$ is the matrix self-energy. We use the conventional notation $\not \partial=\gamma^{\mu} \partial_{\mu}$ and $\not h=\gamma^{\mu} a_{\mu}$ for any four-vector $a^{\mu}$. To simplify formulas, here and in what follows integration over "primed" variables is implied.

Using the canonical form (2) of $G(\underline{1} \underline{2})$, equations (6) can be reduced into equations for the retarded/advanced matrix propagators

$$
\begin{aligned}
& \left(\mathrm{i} \not \partial_{1}-e \not A(\underline{1})-m\right) G^{ \pm}(12)=I \delta(1-2)+\Sigma^{ \pm}\left(11^{\prime}\right) G^{ \pm}\left(1^{\prime} 2\right) \\
& G^{ \pm}(12)\left(-\mathrm{i} \overleftarrow{\not \partial}_{2}-e \not A(\underline{2})-m\right)=I \delta(1-2)+G^{ \pm}\left(11^{\prime}\right) \Sigma^{ \pm}\left(1^{\prime} 2\right)
\end{aligned}
$$

and the Kadanoff-Baym (KB) equations for the correlation functions

$$
\begin{aligned}
& \left(\mathrm{i} \not \partial_{1}-e \not A(1)-m\right) G^{\gtrless}(12)=\Sigma^{+}\left(11^{\prime}\right) G^{\gtrless}\left(1^{\prime} 2\right)+\Sigma^{\gtrless}\left(11^{\prime}\right) G^{-}\left(1^{\prime} 2\right), \\
& G^{\gtrless}(12)\left(-\mathrm{i} \overleftarrow{\not}_{2}-e \not A(2)-m\right)=G^{\gtrless}\left(11^{\prime}\right) \Sigma^{-}\left(1^{\prime} 2\right)+G^{+}\left(11^{\prime}\right) \Sigma^{\gtrless}\left(1^{\prime} 2\right) .
\end{aligned}
$$

Equations (7) and (8) are the starting point in the Green's function approach to QED plasmas [3].

\section{Quasiparticle and off-shell parts of correlation functions}

\subsection{Wigner representation}

It is convenient to transform equations $(7)$ and (8) to the four-dimensional Wigner representation which is defined for any space-time function $F(12)=F\left(x_{1}, x_{2}\right)$ as

$$
F(X, p)=\int \mathrm{d}^{4} x \mathrm{e}^{\mathrm{i} p \cdot x} F(X+x / 2, X-x / 2),
$$

where $p \cdot x=p^{\mu} x_{\mu}=p^{0} t-\boldsymbol{p} \cdot \boldsymbol{r}$. Kinetic description of QED plasmas is based on the assumption that variations in the variable $X$ are slow relative to the scale of $1 / E, 1 /|\boldsymbol{p}|$, where $E$ and $|\boldsymbol{p}|$ are respectively some characteristic particle energy and momentum. Thus, going over to the Wigner representation, the variability of $x$ may be treated perturbatively by expanding all functions in powers of $x$. This leads to the so-called "gradient approximations". We shall restrict our consideration to linear corrections in $X$-gradients. Then, under the Wigner transformation (1),

$$
F_{1}\left(11^{\prime}\right) F_{2}\left(1^{\prime} 2\right) \longrightarrow F_{1}(X, p) F_{2}(X, p)-\frac{i}{2}\left\{F_{1}(X, p), F_{2}(X, p)\right\}
$$

where

$$
\left\{F_{1}(X, p), F_{2}(X, p)\right\}=\frac{\partial F_{1}}{\partial X^{\mu}} \frac{\partial F_{2}}{\partial p_{\mu}}-\frac{\partial F_{1}}{\partial p^{\mu}} \frac{\partial F_{2}}{\partial X_{\mu}}
$$

is the four-dimensional Poisson bracket [3]. It should be noted that some care is required when dealing with spinor-dependent (matrix) quantities since, in general, they do not commute and, consequently, their Poisson brackets do not have the symmetry properties that greatly simplify the 
analysis when Green's functions and self-energies are scalars. Nevertheless, in the following it will prove convenient to use the identities

$$
\begin{aligned}
& A\left\{A^{-1}, A\right\}=\left\{A, A^{-1}\right\} A, \\
& \operatorname{tr}\{A, B\}=-\operatorname{tr}\{B, A\}, \\
& \operatorname{tr}\{A, B\}=-\operatorname{tr}\left\{A^{-1}, A B A\right\},
\end{aligned}
$$

which are valid for any matrices $A(X, p), B(X, p)$ and follow directly from the definition $(3)$ of the Poisson bracket.

\subsection{The electron propagators}

In the Wigner representation, equations (7) for the propagators read

$$
\begin{aligned}
& \left(g^{ \pm}\right)^{-1}(X, p) G^{ \pm}(X, p)=I+\frac{i}{2}\left\{\left(g^{ \pm}\right)^{-1}(X, p), G^{ \pm}(X, p)\right\}, \\
& G^{ \pm}(X, p)\left(g^{ \pm}\right)^{-1}(X, p)=I+\frac{i}{2}\left\{G^{ \pm}(X, p),\left(g^{ \pm}\right)^{-1}(X, p)\right\}
\end{aligned}
$$

where

$$
g^{ \pm}(X, p)=\frac{1}{\not I(X, p)-m-\Sigma^{ \pm}(X, p)}
$$

are the local propagators, and $\Pi^{\mu}(X, p)=p^{\mu}-e A^{\mu}(X)$. To first order in $X$-gradients, from equations (7) one readily derives two "explicit" expressions:

$$
\begin{aligned}
& G^{ \pm}(X, p)=g^{ \pm}+\frac{i}{2} g^{ \pm}\left\{\left(g^{ \pm}\right)^{-1}, g^{ \pm}\right\} \\
& G^{ \pm}(X, p)=g^{ \pm}+\frac{i}{2}\left\{g^{ \pm},\left(g^{ \pm}\right)^{-1}\right\} g^{ \pm} .
\end{aligned}
$$

These are actually equivalent to each other due to the identity (4). Note the appearance of the gradient terms in equations (9), which are absent in the case that propagators are scalar quantities and hence $\left\{\left(g^{ \pm}\right)^{-1}, g^{ \pm}\right\}=\left\{g^{ \pm},\left(g^{ \pm}\right)^{-1}\right\}=0$. The gradient corrections to the propagators may be neglected in evaluating local quantities, say, collision terms in a kinetic equation.

The local propagators (8) have in general a very complicated spinor structure due to the presence of the matrix self-energies $\Sigma^{ \pm}(X, p)$ which may be decomposed into the scalar $(S)$, vector $(V)$, pseudo-scalar $(P)$, axial-vector $(A)$, and tensor $(T)$ components according to [11]

$$
\Sigma^{ \pm}(X, p)=I \Sigma_{(S)}^{ \pm}+\gamma_{\mu} \Sigma_{(V)}^{ \pm \mu}+\gamma_{5} \Sigma_{(P)}^{ \pm}+\gamma_{5} \gamma_{\mu} \Sigma_{(A)}^{ \pm \mu}+\frac{1}{2} \sigma_{\mu \nu} \Sigma_{(T)}^{ \pm \mu \nu}
$$

The situation is improved a great deal, however, for equal probabilities of the spin polarization. In this case a reasonable approximation for the self-energies is $[8,12]$

$$
\Sigma^{ \pm}(X, p)=I \Sigma_{(S)}^{ \pm}(X, p)+\gamma_{\mu} \Sigma_{(V)}^{ \pm \mu}(X, p)
$$

where

$$
\left(\Sigma_{(S)}^{+}\right)^{*}=\Sigma_{(S)}^{-}, \quad\left(\Sigma_{(V)}^{+\mu}\right)^{*}=\Sigma_{(V)}^{-\mu} .
$$

Expression (8) then takes the form

$$
g^{ \pm}(X, p)=\frac{P^{ \pm}+M^{ \pm}}{\left(P^{ \pm}\right)^{2}-\left(M^{ \pm}\right)^{2}}, \quad P^{ \pm \mu}=\Pi^{\mu}-\Sigma_{(V)}^{ \pm \mu}, \quad M^{ \pm}=m+\Sigma_{(S)}^{ \pm} .
$$




\subsection{Decomposition of correlation functions}

Let us now turn to the KB equations (8) in the Wigner representation. Using the transformation rule (2) and keeping only first-order terms in $X$-gradients, we get (arguments $X$ and $p$ are omitted in all functions for brevity)

$$
\begin{aligned}
& \frac{1}{2}\left\{\left(g^{+}\right)^{-1}, G^{\gtrless}\right\}-\frac{1}{2}\left\{\Sigma^{\gtrless}, g^{-}\right\}=i\left(\Sigma^{\gtrless} G^{-}-\left(g^{+}\right)^{-1} G^{\gtrless}\right), \\
& \frac{1}{2}\left\{G^{\gtrless},\left(g^{-}\right)^{-1}\right\}-\frac{1}{2}\left\{g^{+}, \Sigma^{\gtrless}\right\}=i\left(G^{+} \Sigma^{\gtrless}-G^{\gtrless}\left(g^{-}\right)^{-1}\right) .
\end{aligned}
$$

Here one must be careful to distinguish between the full propagators $G^{ \pm}(X, p)$ and the local propagators $g^{ \pm}(X, p)$ in the right-hand sides since equations (9) contain the gradient corrections.

The generalized transport equations for the correlation functions $G^{\gtrless}(X, p)$ are obtained by taking the difference of equations (14). With expressions (8) for the local propagators and relations

$$
\begin{aligned}
& G^{>}(X, p)-G^{<}(X, p)=G^{+}(X, p)-G^{-}(X, p), \\
& \Sigma^{>}(X, p)-\Sigma^{<}(X, p)=\Sigma^{+}(X, p)-\Sigma^{-}(X, p),
\end{aligned}
$$

a simple algebra gives

$$
\begin{aligned}
& \frac{1}{2}\left(\left\{\left(g^{+}\right)^{-1}, G^{\gtrless}\right\}-\left\{G^{\gtrless},\left(g^{-}\right)^{-1}\right\}\right)+\frac{1}{2}\left(\left\{g^{+}, \Sigma^{\gtrless}\right\}-\left\{\Sigma^{\gtrless}, g^{-}\right\}\right) \\
& =-\left[g, \Sigma^{\gtrless}\right]_{-}-\left[\not I-m-\sigma, G^{\gtrless}\right]_{-}+\frac{i}{2}\left(\left[\Sigma^{>}, G^{<}\right]_{+}-\left[\Sigma^{<}, G^{>}\right]_{+}\right),
\end{aligned}
$$

where $[A, B]_{\mp}=A B \mp B A$ stands for the commutator/anticommutator of spinor matrices, and we have introduced the designations

$$
g=\frac{1}{2}\left(G^{+}+G^{-}\right), \quad \sigma=\frac{1}{2}\left(\Sigma^{+}+\Sigma^{-}\right) .
$$

By multiplying equation (16) with the matrices $I, \gamma_{\mu}, \gamma_{5}, \gamma_{5} \gamma_{\mu}, \sigma_{\mu \nu}$, and then taking the trace of all these equations, one finds in general a very complicated set of coupled equations for the components of the correlation functions defined through the spinor decomposition analogous to equation (10). Let us consider one of these transport equations, which is obtained from equation (16) by taking the trace in both sides. The trace of the second drift term is conveniently rearranged as follows:

$$
\begin{aligned}
\operatorname{tr}\left(\left\{g^{+}, \Sigma^{\gtrless}\right\}-\left\{\Sigma^{\gtrless}, g^{-}\right\}\right) & =\operatorname{tr}\left(\left\{g^{+}+g^{-}, \Sigma^{\gtrless}\right\}\right) \\
& =-\operatorname{tr}\left(\left\{\left(g^{+}\right)^{-1}, g^{+} \Sigma^{\gtrless} g^{+}\right\}+\left\{\left(g^{-}\right)^{-1}, g^{-} \Sigma^{\gtrless} g^{-}\right\}\right),
\end{aligned}
$$

where we have used identities (5) and (6). Then the trace of equation (16) may be put in the form

$$
\begin{aligned}
\operatorname{tr}\{\not I I-m-\sigma, & \left.G^{\gtrless}-\frac{1}{2}\left(g^{+} \Sigma^{\gtrless} g^{+}+g^{-} \Sigma^{\gtrless} g^{-}\right)\right\} \\
& +\frac{1}{4} \operatorname{tr}\left\{\Delta \Sigma, g^{+} \Sigma^{\gtrless} g^{+}-g^{-} \Sigma^{\gtrless} g^{-}\right\}=\mathrm{i} \operatorname{tr}\left(\Sigma^{>} G^{<}-\Sigma^{<} G^{>}\right),
\end{aligned}
$$

where $\Delta \Sigma=\Sigma^{+}-\Sigma^{-}$. Let us now write $G^{\gtrless}(X, p)$ as

$$
G^{\gtrless}(X, p)=\widetilde{G}^{\gtrless}+\frac{1}{2}\left(g^{+} \Sigma^{\gtrless} g^{+}+g^{-} \Sigma^{\gtrless} g^{-}\right),
$$


where $\widetilde{G}^{\gtrless}(X, p)$ are some new spinor functions. Substituting this expression into equation (18), it is easy to see that the second term does not contribute to the right-hand side, and we get a transport equation where only the $\widetilde{G}^{\gtrless}(X, p)$ appear:

$$
\operatorname{tr}\left\{\not I I-m-\sigma, \widetilde{G}^{\gtrless}\right\}+\frac{1}{4} \operatorname{tr}\left\{\Delta \Sigma, g^{+} \Sigma^{\gtrless} g^{+}-g^{-} \Sigma^{\gtrless} g^{-}\right\}=\mathrm{i} \operatorname{tr}\left(\Sigma^{>} \widetilde{G}^{<}-\Sigma^{<} \widetilde{G}^{>}\right) .
$$

Decomposition (19) of the correlation functions is closely similar to that proposed previously by Špička and Lipavský [6,7] in non-relativistic kinetic theory. These authors argued that, in the case of small damping, the terms like $\widetilde{G}^{\gtrless}(X, p)$ may be interpreted as "quasiparticle" parts which are sharply peaked near the mass shell, while the additional term represents off-shell (short-time) parts of correlation functions. Within this interpretation, a transport equation for $\widetilde{G}^{<}(X, p)$ can be transformed into a quasiparticle kinetic equation. On the other hand, the off-shell parts of the correlation functions contribute to scattering cross sections [6,7]. It is reasonable to assume that the representation (19) of the electron correlation functions in QED plasmas has the same meaning as in the non-relativistic case. Some arguments that support this assumption will be given below.

\subsection{The full and quasiparticle spectral functions}

In order to exhibit the content of equation (19), it is instructive to consider the spectral properties of $\widetilde{G}^{\gtrless}(X, p)$. We recall that the full spectral function in spinor space is defined as $[8,12]$

$$
\mathcal{A}(X, p)=\mathrm{i}\left(G^{>}(X, p)-G^{<}(X, p)\right)=\mathrm{i}\left(G^{+}(X, p)-G^{-}(X, p)\right) .
$$

In the local approximation, $G^{ \pm}(X, p)=g^{ \pm}(X, p)$, we may use expression (8) to rewrite this spectral function in the form

$$
\mathcal{A}(X, p)=\mathrm{i} g^{+} \Delta \Sigma g^{-}
$$

where

$$
\Delta \Sigma(X, p)=\Sigma^{+}(X, p)-\Sigma^{-}(X, p)
$$

We now introduce the quasiparticle spectral function associated with spinor matrices $\widetilde{G}^{\gtrless}(X, p)$ :

$$
\widetilde{\mathcal{A}}(X, p)=\mathrm{i}\left(\widetilde{G}^{>}(X, p)-\widetilde{G}^{<}(X, p)\right) .
$$

With the aid of equations (19) we obtain the relation between $\widetilde{\mathcal{A}}(X, p)$ and $\mathcal{A}(X, p)$ :

$$
\widetilde{\mathcal{A}}=\mathcal{A}-\frac{\mathrm{i}}{2}\left(g^{+} \Delta \Sigma g^{+}+g^{-} \Delta \Sigma g^{-}\right) .
$$

Recalling equation (22), a little algebra leads to

$$
\widetilde{\mathcal{A}}(X, p)=-\frac{\mathrm{i}}{2} g^{+} \Delta \Sigma g^{+} \Delta \Sigma g^{-} \Delta \Sigma g^{-} .
$$

To illustrate some essential differences between spectral functions (22) and (26), let us consider the zero damping limit. In this special case the local propagators (8) may be replaced by the free particle propagators ${ }^{2}$

$$
g_{0}^{ \pm}(X, p)=\frac{1}{\not I-m \pm \mathrm{i} \not \subset},
$$

where $\varepsilon^{\mu}=(\varepsilon, 0,0,0), \varepsilon \rightarrow+0$. The corresponding retarded/advanced self-energies are $\Sigma^{ \pm}=$ $\mp \mathrm{i} \varepsilon \gamma^{0}$. After some spinor algebra the prelimit expressions for the spectral functions (22) and (26)

\footnotetext{
${ }^{2}$ Strictly speaking, even in the zero damping limit there are corrections to the particle energies coming from the real parts of the retarded/advanced self-energies. However, in a relativistic plasma, which is always weakly coupled, these corrections are very small and may be neglected.
} 
are found to be

$$
\begin{aligned}
& \mathcal{A}(X, p)=\frac{4 \Pi_{0} \Gamma}{\left(\Pi_{0}^{2}-\mathcal{E}_{p}^{2}\right)^{2}+\left(\Pi_{0} \Gamma\right)^{2}}\left[\not \Pi+m+\frac{\mathcal{E}_{p}^{2}-\Pi_{0}^{2}}{2 \Pi_{0}} \gamma^{0}\right], \\
& \widetilde{\mathcal{A}}(X, p)=\frac{4\left(\Pi_{0} \Gamma\right)^{3}}{\left[\left(\Pi_{0}^{2}-\mathcal{E}_{p}^{2}\right)^{2}+\left(\Pi_{0} \Gamma\right)^{2}\right]^{2}}\left[\frac{\mathcal{E}_{p}^{2}+\Pi_{0}^{2}}{2 \Pi_{0}^{2}}(\not I+m)+\frac{\mathcal{E}_{p}^{2}-\Pi_{0}^{2}}{2 \Pi_{0}}\left(1+\frac{\mathcal{E}_{p}^{2}-\Pi_{0}^{2}}{4 \Pi_{0}^{2}}\right) \gamma^{0}\right],
\end{aligned}
$$

where $\mathcal{E}_{p}(X)=\sqrt{\boldsymbol{\Pi}^{2}+m^{2}}$ and $\Gamma=2 \varepsilon$ is the infinitesimally small damping width.

From the above expressions two important observations can be made. First, one easily verifies that both spectral functions have the same limiting form:

$$
\lim _{\Gamma \rightarrow 0} \mathcal{A}=\lim _{\Gamma \rightarrow 0} \widetilde{\mathcal{A}}=2 \pi \eta\left(\Pi_{0}\right) \delta\left(\Pi^{2}-m^{2}\right)(\not \Pi+m),
$$

where $\eta\left(\Pi_{0}\right)=\Pi_{0} /\left|\Pi_{0}\right|$. Second, it is seen that the prefactor in $\widetilde{\mathcal{A}}$ approaches the delta function faster than the prefactor in $\mathcal{A}$. Thus, in the case of small damping, it is reasonable to suggest that the term $\widetilde{G}^{\gtrless}$ in equation (19) may be expressed to close approximation by a singular mass-shell form, while the last term may be regarded as the off-shell contribution to the correlation functions.

\subsection{The pole approximation}

We now want to find a way of expressing $\widetilde{G}^{\gtrless}(X, p)$ in terms of the electron and positron distribution functions, which is required to convert equation (16) into a kinetic equation and calculate the scattering rates in a physically transparent form.

Let us first consider the hermicity properties of $\widetilde{G}^{\gtrless}(X, p)$ and $\widetilde{A}(X, p)$. Note that the correlation functions $G^{\gtrless}(X, p)$, propagators $G^{ \pm}(X, p)$, and the self-energies, as spinor matrices, satisfy relations

$$
\begin{array}{ll}
{\left[G^{\gtrless}(X, p)\right]^{\dagger}=-\gamma^{0} G^{\gtrless}(X, p) \gamma^{0},} & {\left[\Sigma^{\gtrless}(X, p)\right]^{\dagger}=-\gamma^{0} \Sigma^{\gtrless}(X, p) \gamma^{0},} \\
{\left[G^{+}(X, p)\right]^{\dagger}=\gamma^{0} G^{-}(X, p) \gamma^{0},} & {\left[\Sigma^{+}(X, p)\right]^{\dagger}=\gamma^{0} \Sigma^{-}(X, p) \gamma^{0},}
\end{array}
$$

which follow from the definition of these quantities. Recalling (8), it is easy to see that the local propagators $g^{ \pm}(X, p)$ satisfy the same relations as $G^{ \pm}(X, p)$. Then one derives from equation (19)

$$
\left[\widetilde{G}^{\gtrless}(X, p)\right]^{\dagger}=-\gamma^{0} \widetilde{G}^{\gtrless}(X, p) \gamma^{0} .
$$

This immediately leads to the following property of the quasiparticle spectral function (24):

$$
[\widetilde{\mathcal{A}}(X, p)]^{\dagger}=\gamma^{0} \widetilde{\mathcal{A}}(X, p) \gamma^{0}
$$

We now introduce the distribution functions in spinor space, $\mathcal{F}^{\gtrless}(X, p)$, through

$$
\widetilde{G}^{\gtrless}(X, p)=\mp \frac{i}{2}\left(\widetilde{\mathcal{A}} \mathcal{F}^{\gtrless}+\mathcal{F}^{\gtrless} \widetilde{\mathcal{A}}\right), \quad \mathcal{F}^{>}(X, p)+\mathcal{F}^{<}(X, p)=I .
$$

Relation (24) is automatically satisfied, whereas equations (32) and (33) require that

$$
\left[\mathcal{F}^{\gtrless}(X, p)\right]^{\dagger}=\gamma^{0} \mathcal{F}^{\gtrless}(X, p) \gamma^{0} \text {. }
$$

In particular, from this property it follows that in the spinor decomposition of $\mathcal{F}^{\gtrless}(X, p)$ [cf. (10)] all coefficients are real. 
The spinor structure of $\mathcal{F}^{\gtrless}(X, p)$ is in general very complicated. For a weakly coupled plasma, however, reasonable approximations may be introduced by starting from the case of non-interacting particles, where $G^{\gtrless}(X, p)=\widetilde{G}^{\gtrless}(X, p)$ and the correlation functions are expressed in terms of the electron (positron) distribution functions by using the plane-wave expansion of the field operators [11]. Another way of arriving at the same result is to consider the sum of KB equations (14) in the collisionless and slow variation limit [3]:

$$
\left[(\not I-m), \widetilde{G}^{\gtrless}\right]_{+}=0 .
$$

Then, using equations (30), (34), and the spinor decomposition of $\widetilde{\mathcal{F}}^{\gtrless}$, it can be shown that

$$
\mathcal{F}^{\gtrless}(X, p)=\frac{1}{2} \sum_{s}\left(I+\gamma_{5} \phi\right) F_{s}^{\gtrless}(X, p)
$$

with $s^{\mu}$ satisfying $s^{\mu} s_{\mu}=-1$ and $s^{\mu} \Pi_{\mu}=0$. In the rest frame $s^{\mu}=(0, s)$, where the unit vector $s$ represents the direction of spin. As discussed by Bezzerides and DuBois [3], the quantities $F_{s}^{\gtrless}(X, p)$ are related directly to the electron (positron) distribution functions for different spin states ${ }^{3}$. In the case of equal probabilities of the spin polarization equation (37) reduces to

$$
\mathcal{F}^{\gtrless}(X, p)=I F^{\gtrless}(X, p),
$$

where

$$
F^{\gtrless}(X, p)=\frac{1}{2} \sum_{s} F_{s}^{\gtrless}(X, p), \quad F^{>}(X, p)+F^{<}(X, p)=1 .
$$

Then the correlation functions in the collisionless limit ${ }^{4}$ are given by

$$
\widetilde{G}^{\gtrless}(X, p)=\mp 2 \pi \mathrm{i} \eta\left(\Pi^{0}\right) \delta\left(\Pi^{2}-m^{2}\right)(\not I+m) F^{\gtrless}(X, p) .
$$

Before going further, one remark is appropriate here. The appearance of the vector potential $A^{\mu}(X)$ in equation (40) and other formulas is due to the fact that the electron Green's function (5) and, consequently, the propagators $G^{ \pm}(12)$ and the correlation functions $G^{\gtrless}(12)$ are not invariant under gauge transformation of the mean electromagnetic field. In principle, we could work from the beginning with the gauge invariant Green's function defined as

$$
\mathcal{G}(\underline{1} \underline{2})=G(\underline{1} \underline{2}) \exp \left[\mathrm{i} e \int_{\underline{2}}^{\underline{1}} A_{\mu}(\underline{x}) \mathrm{d} \underline{x}^{\mu}\right],
$$

where $\underline{x}^{\mu}=(\underline{t}, \boldsymbol{r})$ and integration over $\boldsymbol{r}$ is performed along a straight line connecting the points $\boldsymbol{r}_{2}$ and $\boldsymbol{r}_{1}$. It is an easy matter, however, to pass to the gauge invariant functions, $\mathcal{G}^{ \pm}(X, p)$ and $\mathcal{G}^{\gtrless}(X, p)$, in the Wigner representation. Within the first-order gradient approximation the corresponding relations are

$$
\mathcal{G}^{ \pm}(X, p)=G^{ \pm}(X, p+e A(X)), \quad \mathcal{G}^{\gtrless}(X, p)=G^{\gtrless}(X, p+e A(X)) .
$$

For instance, from equation (40) we see that the gauge invariant quasiparticle correlation functions in the collisionless limit are given by

$$
\widetilde{\mathcal{G}}^{\gtrless}(X, p)=\mp 2 \pi \mathrm{i} \eta\left(p^{0}\right) \delta\left(p^{2}-m^{2}\right)(\not p+m) f^{\gtrless}(X, p),
$$

where $f^{\gtrless}(X, p)=F^{\gtrless}(X, p+e A(X))$ are the gauge invariant distribution functions.

\footnotetext{
${ }^{3}$ Note that expressions for the correlation functions in the collisionless limit given in [3] are not quite correct. They correspond to the form $\widetilde{G}^{\gtrless}=\mp \mathrm{i} \widetilde{\mathcal{A}} \mathcal{F}^{\gtrless}$ and, if matrices $\mathcal{F}^{\gtrless}$ are non-diagonal, violate the property (32) in contrast to the symmetric representation (34).

${ }^{4}$ We recall that in the case being considered $\widetilde{G}^{\gtrless}=G^{\gtrless}$.
} 
Since in a weakly coupled (nearly collisionless) plasma the difference between the free particle energy and the quasiparticle energy may be neglected, the expression (40) is a reasonable approximation for the first (quasiparticle) term in equation (19). The pole approximation (40) was previously proposed by Bezzerides and DuBois [3], although for the full correlation functions $G^{\gtrless}$, so that the off-shell term in equation (19) was missing.

\subsection{Extended quasiparticle approximation for the correlation functions}

Insertion of (40) into equation (20) leads to a kinetic equation for the electron $\left(f_{-}\right)$and positron $\left(f_{+}\right)$distribution functions defined as

$$
\left.f^{<}(X, p)\right|_{p^{0}=E_{p}}=f_{-}(X, \boldsymbol{p}),\left.\quad f^{<}(X, p)\right|_{p^{0}=-E_{p}}=1-f_{+}(X,-\boldsymbol{p}),
$$

where $E_{p}=\sqrt{\boldsymbol{p}^{2}+m^{2}}$. Neglecting unessential self-energy corrections to the drift, we arrive at the kinetic equation which is formally the same as that derived by Bezzerides and DuBois [3]. The new feature is that now the additional off-shell parts of the correlation functions (19) contribute to the scattering cross sections in the collision term.

To discuss this point we recall the general expression for the matrix self-energy $\Sigma(\underline{1} \underline{2})$ (see, e.g., [13]). In the Coulomb gauge it can be written as

$$
\Sigma(\underline{1} \underline{2})=-\gamma^{0} G\left(\underline{1} \underline{1}^{\prime}\right) \Gamma^{L}\left(\underline{1}^{\prime} \underline{2} ; \underline{2}^{\prime}\right) D^{L}\left(\underline{2}^{\prime} \underline{1}\right)-\gamma^{i} G\left(\underline{1} \underline{1}^{\prime}\right) \Gamma_{j}^{T}\left(\underline{1}^{\prime} \underline{2} ; \underline{2}^{\prime}\right) D_{j i}^{T}\left(\underline{2}^{\prime} \underline{1}\right),
$$

where $\Gamma^{L}$ and $\Gamma_{j}^{T}$ are, respectively, the longitudinal and transverse vertex functions, $D^{L}(\underline{1} \underline{2})$ is the longitudinal (plasmon) Green's function

$$
D^{L}(\underline{1} \underline{2})=\mathrm{i}\left\langle T_{C} \Delta \hat{\phi}(\underline{1}) \Delta \hat{\phi}(\underline{2})\right\rangle-\frac{1}{4 \pi\left|\boldsymbol{r}_{1}-\boldsymbol{r}_{2}\right|} \delta\left(\underline{t}_{1}-\underline{t}_{2}\right)
$$

with $\Delta \hat{\phi}(\underline{1})=\hat{\phi}(\underline{1})-\phi(\underline{1})$, and $D_{i j}^{T}(\underline{1} \underline{2})$ is the transverse photon Green's function

$$
D_{i j}^{T}(\underline{1} \underline{2})=-\mathrm{i}\left\langle T_{C} \Delta \hat{A}_{i}(\underline{1}) \Delta \hat{A}_{j}(\underline{2})\right\rangle
$$

with $\Delta \hat{A}_{i}(\underline{1})=\hat{A}_{i}(\underline{1})-A_{i}(\underline{1})$. Using the canonical representation (2), equation (45) can be written for the components $\Sigma^{\gtrless}(12)$. Since the resulting expression is rather complicated, we shall restrict the discussion to the case where the full vertex functions are replaced by the bare ones

$$
\begin{aligned}
& \Gamma^{L(0)}(\underline{1} \underline{2} ; \underline{3})=\mathrm{i} e^{2} \delta(\underline{1}-\underline{2}) \delta(\underline{1}-\underline{3}) \gamma^{0}, \\
& \Gamma_{i}^{T(0)}(\underline{1} \underline{2} ; \underline{3})=-\mathrm{i} e^{2} \delta(\underline{1}-\underline{2}) \delta_{i k}^{T}(\underline{1}-\underline{3}) \gamma^{k},
\end{aligned}
$$

where $\delta_{i k}^{T}(\underline{1}-\underline{3})$ is the transverse delta function on the contour $C$ :

$$
\delta_{i j}^{T}(\underline{1}-\underline{2})=\delta\left(\underline{t}_{1}-\underline{t}_{2}\right) \int \frac{\mathrm{d}^{3} \boldsymbol{k}}{(2 \pi)^{3}} \mathrm{e}^{\mathrm{i} \boldsymbol{k} \cdot\left(\boldsymbol{r}_{1}-\boldsymbol{r}_{2}\right)}\left(\delta_{i j}-\frac{k_{i} k_{j}}{\boldsymbol{k}^{2}}\right) .
$$

With expressions (48) for the vertex functions, equation (45) becomes

$$
\Sigma(\underline{1} \underline{2})=-\mathrm{i} e^{2} \gamma^{0} G(\underline{1} \underline{2}) \gamma^{0} D^{L}(\underline{2} \underline{1})+\mathrm{i} e^{2} \gamma^{i} G(\underline{1} \underline{2}) \gamma^{j} D_{j i}^{T}{ }^{\lessgtr}(\underline{2} \underline{1}) .
$$

For the components $\Sigma^{\gtrless}(12)$ this reads

$$
\Sigma^{\gtrless}(12)=-\mathrm{i} e^{2} \gamma^{0} G^{\gtrless}(12) \gamma^{0} D^{L \lessgtr}(21)+\mathrm{i} e^{2} \gamma^{i} G^{\gtrless}(12) \gamma^{j} D_{j i}^{T}(21) .
$$

We see that equation (19) itself does not determine the correlation functions in terms of the distribution functions because the self-energies $\Sigma^{\gtrless}$ depend on $G^{\gtrless}$. 
Let us now make the Wigner transformation in equation (51) and introduce the principal-axis representation for photon states through [3]

$$
D_{i j}^{T \gtrless}(X, k)=\sum_{s} \epsilon_{s i}(X, k) d_{s}^{\gtrless}(X, k) \epsilon_{s j}(X, k),
$$

where $\boldsymbol{\epsilon}_{s}(X, k),(s=1,2)$, are unit polarization vectors satisfying

$$
\boldsymbol{\epsilon}_{s}(X, k) \cdot \boldsymbol{\epsilon}_{s^{\prime}}(X, k)=\delta_{s s^{\prime}}, \quad \sum_{s} \epsilon_{s i}(X, k) \epsilon_{s j}(X, k)=\delta_{i j}-\frac{k_{i} k_{j}}{\boldsymbol{k}^{2}} .
$$

Then equation (51) is transformed into ${ }^{5}$

$$
\begin{aligned}
& \Sigma^{\gtrless}(X, p)=-\mathrm{i} e^{2} \int \frac{\mathrm{d}^{4} p^{\prime}}{(2 \pi)^{4}} D^{L^{\lessgtr}}\left(X, p^{\prime}-p\right) \gamma^{0} G^{\gtrless}\left(X, p^{\prime}\right) \gamma^{0} \\
& \quad+\mathrm{i} e^{2} \sum_{s} \int \frac{\mathrm{d}^{4} p^{\prime}}{(2 \pi)^{4}} d_{s}^{\lessgtr}\left(X, p^{\prime}-p\right) \oiint_{s}\left(X, p^{\prime}-p\right) G^{\gtrless}\left(X, p^{\prime}\right) \notin_{s}\left(X, p^{\prime}-p\right),
\end{aligned}
$$

where we have introduced the polarization four-vectors $\epsilon_{s}^{\mu}=\left(0, \boldsymbol{\epsilon}_{s}\right)$. Insertion of (54) into equation (19) leads to the integral equation for the electron correlation functions which may be approximately solved by iteration since the self-energies $\Sigma^{\gtrless}(X, p)$ are proportional to the small coupling constant $^{6}$. Following this way, one obtains the full correlation functions as some functionals of the quasiparticle correlation functions, or, with equation (34), as functionals of the distribution functions in spinor space. These functionals can then be used to obtain from equation (45) an analogous representation for the self-energies $\Sigma^{\gtrless}(X, p)$. Note, however, that some care is required in order that the self-energies be calculated in a self-consistent fashion. The point is that the vertex functions in equation (45) contain higher-order terms in the coupling constant, which must be considered along the off-shell parts of $G^{\gtrless}(X, p)$. Therefore, taking the vertex functions in the form (48), one may keep only the leading off-shell corrections to the correlation functions. Within this approximation, we obtain from equations (19) and (54) (the fixed argument $X$ is omitted everywhere for brevity)

$$
\begin{gathered}
G^{\gtrless}(p)=\widetilde{G}^{\gtrless}(p)-\frac{\mathrm{i} e^{2}}{2} \int \frac{\mathrm{d}^{4} p^{\prime}}{(2 \pi)^{4}} D^{L}\left(p^{\prime}-p\right)\left[g^{+}(p) \gamma^{0} \widetilde{G}^{\gtrless}\left(p^{\prime}\right) \gamma^{0} g^{+}(p)+g^{-}(p) \gamma^{0} \widetilde{G}^{\gtrless}\left(p^{\prime}\right) \gamma^{0} g^{-}(p)\right] \\
+\frac{\mathrm{i} e^{2}}{2} \sum_{s} \int \frac{\mathrm{d}^{4} p^{\prime}}{(2 \pi)^{4}} d_{s}^{\lessgtr}\left(p^{\prime}-p\right)\left[g^{+}(p) \phi_{s}\left(p^{\prime}-p\right) \widetilde{G}^{\gtrless}\left(p^{\prime}\right) \notin_{s}\left(p^{\prime}-p\right) g^{+}(p)\right. \\
\left.+g^{-}(p) \notin_{s}\left(p^{\prime}-p\right) \widetilde{G}^{\gtrless}\left(p^{\prime}\right) \notin_{s}\left(p^{\prime}-p\right) g^{-}(p)\right] .
\end{gathered}
$$

Keeping here only the first (quasiparticle) contribution and then taking the simplest pole form (40), we recover in fact for the self-energies (54) the expression which was used by Bezzerides and DuBois [3] to calculate the collision term in equation (20). As already mentioned in the introduction, this quasiparticle approximation is sufficient to describe electron-electron (electronpositron) scattering and Cherenkov emission/absorption of longitudinal plasma waves. Inclusion of the off-shell integral terms in equation (55) leads to the extended quasiparticle approximation

\footnotetext{
${ }^{5}$ In the self-energies we have omitted the gradient corrections which give irrelevant contributions to the drift term in the particle kinetic equation.

${ }^{6}$ It should be noted that, in contrast to vacuum electrodynamics where one deals with an asymptotic expansion in powers of $e^{2}$, the Green's functions for particles and the field in plasmas contain polarization and multiple-scattering effects to all orders on $e^{2}$. As discussed by Bezzerides and DuBois [3], the dimensionless strength of interactions is characterized by the plasma parameter which is always small for relativistic plasmas. Formally, in this case the expansion is performed in terms of the Green's functions $D^{L}$ and $D^{T}$ which characterize the intensity of fluctuations of the electromagnetic field.
} 
for the correlation functions, which is necessary for description of higher-order processes, such as Bremsstrahlung and Compton scattering in plasmas. This requires, however, a more detailed analysis of the vertex functions and the field Green's functions, which will be done in future publications (see also [14]).

\section{Discussion}

We have shown that the decomposition of the electron correlation functions in QED plasmas into the quasiparticle and off-shell parts can be done in a rather general form (19) which bears resemblance to the analogous decomposition in non-relativistic kinetic theory [6,7]. Note that equation (19) itself does not imply from the outset the pole approximation for the quasiparticle parts. Nevertheless, we have seen that the "collisional broadening" of the quasiparticle spectral function (26) is considerably smaller than that of the full spectral function (22). Therefore, at some stage the pole approximation for the quasiparticle terms is sufficient in order to calculate scattering rates in a kinetic equation. It should be emphasized, however, that even in the case of a weakly coupled plasma the pole form (40) is not always an adequate approximation for $\widetilde{G}^{\gtrless}(X, p)$ since it can violate some important properties of the full correlation functions $G^{\gtrless}(X, p)$, say, the sum rules. In such situations improved forms of the quasiparticle spectral function $\widetilde{A}(X, p)$ have to be used in the general representation (34).

\section{Acknowledgement}

This work was supported by "DFG-Sonderforschungsbereich 652" and U.S.CRDF-RF Ministry of Education Award VZ-010-0.

\section{References}

1. Santala M.I.K. et al., Phys. Rev. Lett., 2001, 86, 1227.

2. Mangles S P D. et al., Phys. Rev. Lett., 2005, 94, 245001.

3. Bezzerides B., DuBois D.F., Ann. Phys. (N.Y.), 1972, 70, 10.

4. Köhler H.S., Malfliet R., Phys. Rev. C, 1993, 48, 1034.

5. Bornath Th., Kremp D., Kraeft W.D., Schlanges M., Phys. Rev. E, 1996, 54, 3274.

6. Špička V., Lipavský P., Phys. Rev. Lett., 1994, 73, 3439.

7. Špička V., Lipavský P., Phys. Rev. B, 1995, 52 (20), 14615.

8. Botermans W., Malfliet R., Phys. Rep., 1990, 198, 115.

9. Danielewicz P., Ann. Phys. (N.Y.), 1984, 152, 239.

10. DuBois D. F. - In: Lectures in Theoretical Physics, edited by W.E. Brittin. Gordon and Breach, New York, 1967, 469-619.

11. Itzykson C., Zuber J.-B. Quantum Field Theory. McGraw-Hill, New York, 1980.

12. Mrówczyński St., Heinz U., Ann. Phys. (N.Y.), 1994, 229, 1.

13. Bonitz M. Quantum Kinetic Theory. Teubner, Stuttgart-Leipzig, 1998.

14. Morozov V.G., Röpke G., J. Phys.: Conference Series, 2006, 35, 110. 


\title{
Розширене квазічастинкове наближення для релятивістських електронів у плазмі
}

\author{
В.Г.Морозов ${ }^{1}$, Г.Репке ${ }^{2}$
}

1 Московський державний інститут радіотехніки, електроніки та автоматики, просп. Вернадського 78, 117454 Москва, Росія

2 Університет м.Ростока, фізичний факультет, 18051 Росток, Німеччина

Отримано 17 квітня 2006 р., в остаточному вигляді - 6 червня 2006 р.

\begin{abstract}
Виходячи з рівняння Дайсона для впорядкованої по контуру функції Гріна, показано, що кореляційні функції для релятивістських електронів (позитронів) у слабозв'язаній нерівноважній плазмі можна розкласти на квазічастинкові частини та короткочасові (off-shell) частини у досить загальній формі. 3 точністю до головного порядку по електромагнітній константі взаємодії цей розклад дає розширене квазічастинкове наближення для кореляційних функцій, яке можна використати для розрахунків 3 перших принципів швидкості розсіяння у КЕД плазмі.
\end{abstract}

Ключові слова: багаточастинкова КЕД, нерівноважні функції Гріна, кінетична теорія релятивістської плазми

PACS: 05.30.-d, 52.25.Dg, 52.27.-h, 52.27. Ny 